\title{
Measuring the impact of Verticillium longisporum on oilseed rape (Brassica napus) yield in field trials in the United Kingdom
}

\author{
J. R. L. Depotter • B. P. H. J. Thomma ・ T. A. Wood
}

Accepted: 2 July 2018 / Published online: 8 July 2018

(C) The Author(s) 2018

\begin{abstract}
Verticillium longisporum causes Verticillium stem striping on oilseed rape, which appears towards the end of the cropping season. Thus far, the impact of $V$. longisporum infection on oilseed yield remains unclear. In this study, we assessed the impact of Verticillium stem striping on British oilseed rape production. To this end, four cultivars (Incentive, Vision, Harper and Quartz) were grown in field plots with different levels of $V$. longisporum disease pressure at different locations over two consecutive years. Whereas Incentive and Vision developed relatively few stem striping symptoms, Harper and especially Quartz showed severe symptoms during these field experiments. Furthermore, higher inoculum levels induced more severe symptoms in these cultivars. Significant yield reductions upon $V$. longisporum infection only occurred in a single field trial on all tested oilseed rape cultivars. These preliminary data suggest that Verticillium stem striping does not consistently impact oilseed rape yield, despite the occurrence of abundant disease symptoms.
\end{abstract}

B. P. H. J. Thomma and T. A. Wood contributed equally to this work

J. R. L. Depotter • B. P. H. J. Thomma $(\bowtie)$

Laboratory of Phytopathology, Wageningen University and

Research, Droevendaalsesteeg 1, 6708 PB Wageningen,

The Netherlands

e-mail: bart.thomma@wur.nl

J. R. L. Depotter - T. A. Wood

Department of Crops and Agronomy, National Institute of

Agricultural Botany, Huntingdon Road, Cambridge CB3 OLE, UK
Keywords Verticillium stem striping · Yield loss .

Disease development · Oil content

Verticillium stem striping is an oilseed rape disease caused by the fungal pathogen Verticillium longisporum, one of the ten species of the Verticillium genus (Depotter et al. 2016; Inderbitzin et al. 2011). This disease is well established in north-central Europe (Heale and Karapapa 1999; Steventon et al. 2002) and recently emerged in the United Kingdom for the first time (Depotter et al. 2017b; Gladders et al. 2011). In contrast to other Verticillium spp., V. longisporum generally infects brassicaceous plants (Inderbitzin and Subbarao 2014; Novakazi et al. 2015; Zeise and von Tiedemann 2002). On these hosts, V. longisporum causes wilting symptoms (Koike et al. 1994). However, no wilting symptoms are observed on oilseed rape (Heale and Karapapa 1999). Rather, dark unilateral striping is induced at the end of the cropping season before the onset of maturation, approximately 3-4 weeks before harvest. This coincides with the formation of black microsclerotia by the fungus in the stem cortex underneath the epidermis. There is no effective fungicide treatment to combat Verticillium diseases; hence, using resistant cultivars is the preferred management strategy against Verticillium stem striping (Depotter et al. 2016). Unfortunately, genuine resistance $(R)$ genes are unavailable against $V$. longisporum. Alternatively, research efforts have been undertaken to improve partial Verticillium resistance in oilseed rape (Eynck et al. 2009; Obermeier et al. 2013). Despite these efforts to improve 
resistance in oilseed rape cultivars, limited studies investigated the actual impact of Verticillium stem striping on crop quality and quantity (Dunker et al. 2008). Yield losses by Verticillium stem striping have been anticipated to range from 10 to $50 \%$, yet experimental verifications of such estimations are lacking (Dunker et al. 2008). In contrast, Verticillium stem striping did not impact yield significantly in previous field studies despite the presence of stem striping symptoms (Dunker et al. 2008).

To investigate the impact of Verticillium stem striping on oilseed rape production, we performed field experiments in two consecutive years at different locations in the United Kingdom (UK). In harvest year 2016, field plots were located in Hinxton, Cambridgeshire, UK, whereas in harvest year 2017 fields were situated in Higham, Suffolk, UK. Previously, winter oilseed rape cultivars were screened for their resistance against Verticillium stem striping under field conditions (not published). We selected four commercially used cultivars of this screen for our field experiments: two openpollinated cultivars Vision (Senova) and Quartz (KWS), and two hybrid types: Incentive (DSV) and Harper (Bayer). In the screen, Incentive and Vision displayed little symptoms, whereas Harper and Quartz displayed stem-striping symptoms abundantly. These differences in susceptibility were also confirmed by pathogenicity tests under controlled greenhouse conditions, where Quartz displayed more severe disease symptom development upon $V$. longisporum inoculation compared to Incentive (Depotter et al. 2017a). The field plots were sown 5th and 21st September, for the field trials at Hinxton and Higham, respectively. To monitor putative dosage effects, plots were inoculated with different quantities of $V$. longisporum inoculum. To this end, a mix of British Verticillium strains were cultured in bags containing a sterile, moist medium of vermiculite $(800 \mathrm{ml})$ and maize meal $(26 \mathrm{~g})$ for approximately four weeks and then dried at room temperature for a week prior to inoculation. The plots were inoculated by manually spreading 0.251 or 2.51 of thoroughly mixed, colonized vermiculite/maize meal medium on the soil surface. To enable homogenous dispersion, the 0.251 of inoculum was first mixed with 0.751 sand. A control treatment was left un-inoculated. Inoculations were performed the 16th of September 2015 for the field trial at Hinxton and the 26th of September 2016 for the trial at Higham. As four different cultivars and three different inoculum levels were tested in four replicates, the field trial comprised of 48 plots, which were arranged in a semi-randomized block design and were $24 \mathrm{~m}^{2}(12 \mathrm{~m} \times$ $2 \mathrm{~m}$ ) in size. The trials were managed according common agronomic practices with one fertilizer application in August (diammonium phosphate) and two in February-April $(\mathrm{N}+\mathrm{S}$ and $\mathrm{N}$, respectively). No fungicides and a standard insecticide programme were used (to control flea beetle during establishment and pollen beetle during flowering). Field plots were harvested on 31th and 26th July for the field trials at Hinxton and Higham, respectively. To this end, a central strip of $18 \mathrm{~m}^{2}$ was harvested using a combine and used for analysis.

We assessed the Verticillium stem striping disease symptoms by monitoring severity and incidence. Disease symptom severity was measured based on the fraction of stem circumference that displayed Verticillium stem striping symptoms. For every plot at each time point, 20 stems were randomly chosen and scored on a $0-10$ scale, with each interval representing a $10 \%$ increase in dark unilateral striping of the stem circumference at knee-height (eg. Score $2=20 \%$ striping). Disease incidence was the fraction of the stems containing disease symptoms with a minimal score of 1 . The symptoms were scored four times each year starting from the end of June with a 7-day interval with the first assessment on 30th June and 27th June for year 2016 and 2017, respectively. In this way, area under disease progress curve (AUDPC) could be calculated by trapezoidal integration to compare disease severities over time (Shaner and Finney 1977).

In correspondence to the unpublished cultivar screen, cultivars Incentive and Vision displayed equally few disease symptoms at the end of both cropping seasons (Table 1). Consequently, little to no progress in disease symptoms was monitored in both years (Fig. 1). In contrast, Harper displayed more extensive disease symptoms in both consecutive trial years (Table 1). Altogether, disease symptom development was similar in harvest years 2016 and 2017 (Fig. 1). Higher inoculum levels resulted in increasing incidence and symptom severity in Harper for the 2016 trial (Table 1). However, whereas disease levels of the inoculated plots were higher than in control plots, symptoms levels between the lower and higher inoculum treatments could not be distinguished in 2017 (Table 1). Furthermore, Quartz displayed the most severe disease symptoms of the four tested cultivars upon $V$. longisporum challenge (Table 1). The area under disease progress curves 


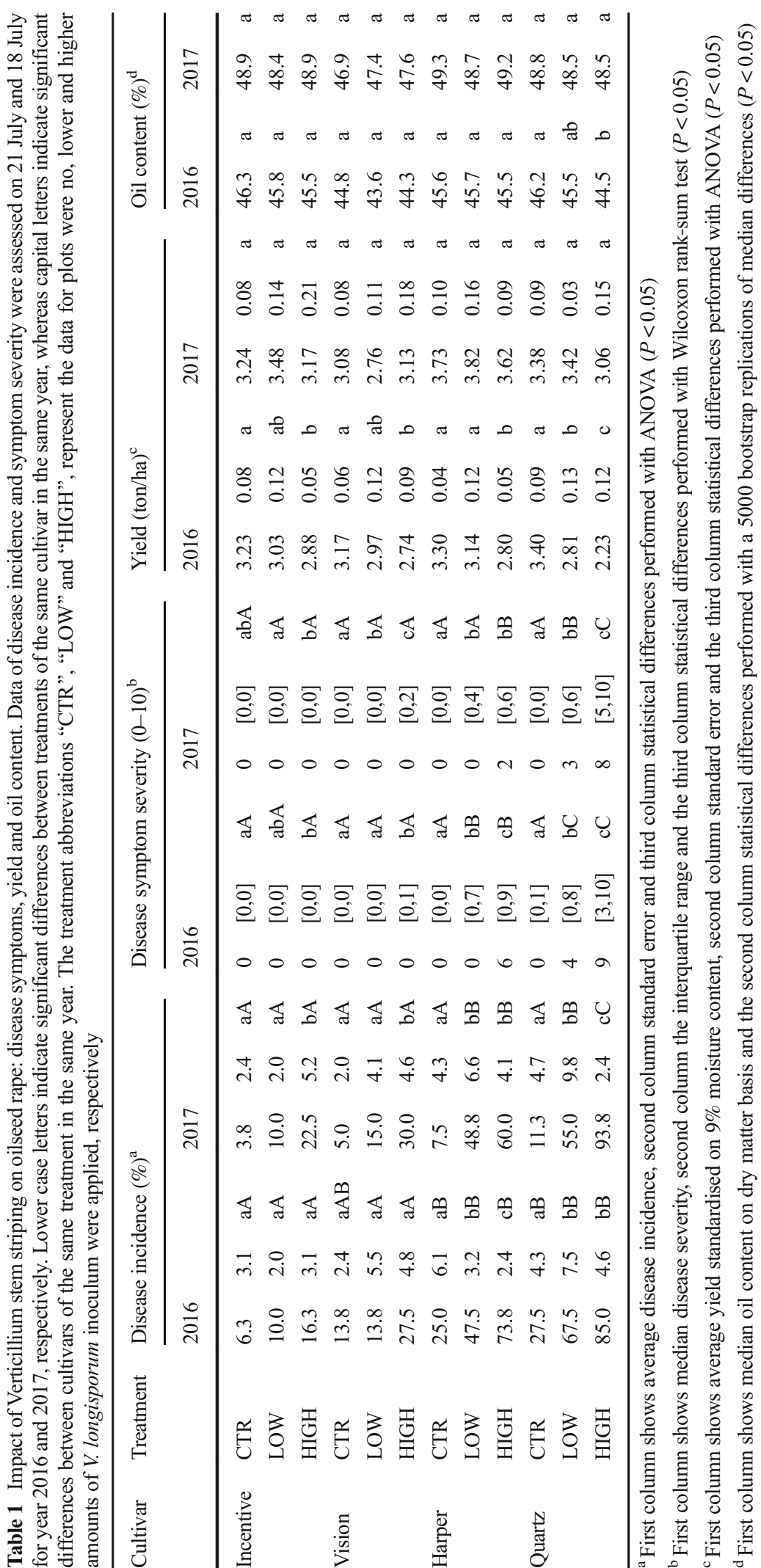


Incentive

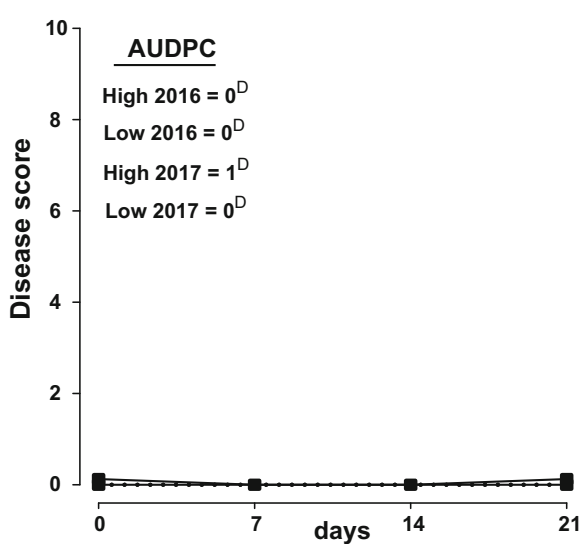

Harper

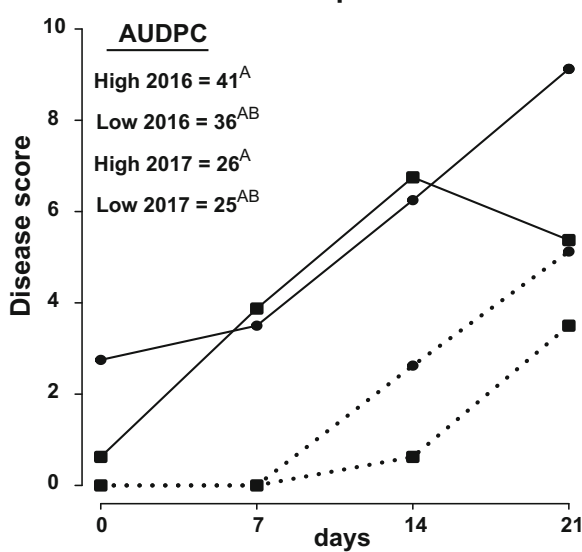

Fig. 1 Verticillium stem striping progression during the end of the cropping season. The Y-axis represents the median of the upper quartile plot disease score. The $\mathrm{X}$-axis represents the number of days after the first scoring: 30th June and 27th June for

(AUDPCs) for the lower and higher inoculum treatments were similar for both years (Fig. 1). Higher inoculum levels resulted in increasing incidence and symptom severity in Quartz for both cropping years (Table 1). Thus, in general, the symptom development of the four cultivars was consistent for the harvest years 2016 and 2017.

In order to evaluate whether the different symptom development between cultivars corresponded with economically important crop parameters, seed yield and oil content were measured. To this end, yield of oilseed rape were adjusted to $9 \%$ moisture content and oil content of the seeds was determined by Nuclear Magnetic Resonance (NMR) spectroscopy (Benchtop NMR Analyser - MQC, Oxford Instruments, UK). Differences in
Vision
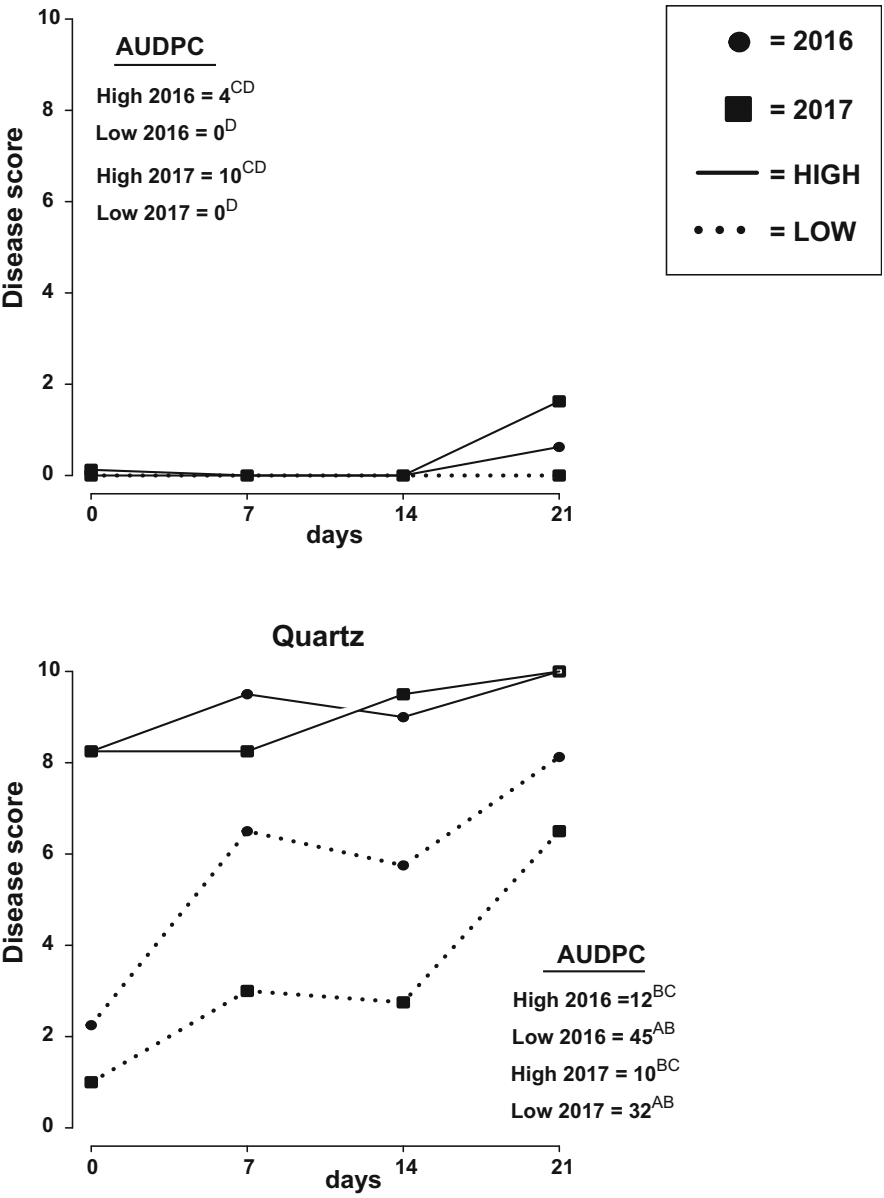

harvest years 2016 and 2017, respectively. The area under progress disease curve (AUPDC) was calculated and letters present significance levels for all panels collectively calculated with 50,000 bootstrap replications of median differences $(P<0.05)$

oilseed rape cultivar and trial year impacted significantly on seed yield and oil content, whereas differences in the level of inoculum only impacted on yield and not oil content (Table 2). However, the impact of inoculum level was inconsistent between the two cropping years as significant differences in yield and oil content could only be found in the 2016 field trial (Table 1). In 2016, plots with the higher inoculum rate yielded significantly less than control plots for all tested oilseed rape cultivars. The highest yield impact was for Quartz that demonstrated a $34 \%$ reduction compared to the uninoculated control, which is within the range of previously made estimates of 10 to $50 \%$ (Table 1) (Dunker et al. 2008). Despite the presence of only mild disease symptoms, the yield in 2016 was also significantly 
Table 2 Analysis of variance for effects on seed yield and oil content. Multivariate ANOVA was used: Yield/Oil content $\sim$ Cultivar + Year + Inoculum level + Repeat

\begin{tabular}{|c|c|c|c|c|c|c|c|c|c|c|}
\hline & \multicolumn{5}{|c|}{ Yield } & \multicolumn{5}{|c|}{ Oil content } \\
\hline & Df & Sum $\mathrm{Sq}$ & Mean Sq & $F$-value & $P$-value & Df & Sum Sq & Mean Sq & $F$-value & $P$-value \\
\hline \multicolumn{11}{|l|}{ Main effects } \\
\hline A: Cultivar & 3 & 2.53 & 0.84 & 10.19 & $8.28 \times 10^{-6}$ & 3 & 33.81 & 11.27 & 16.21 & $1.95 \times 10^{-8}$ \\
\hline B: Year & 1 & 2.93 & 2.93 & 35.39 & $5.66 \times 10^{-8}$ & 1 & 253.27 & 253.27 & 364.32 & $<2 \times 10^{-16}$ \\
\hline C: Inoculum level & 2 & 2.14 & 1.07 & 12.94 & $1.22 \times 10^{-5}$ & 2 & 1.19 & 0.60 & 0.86 & 0.43 \\
\hline D: Repeat & 3 & 0.38 & 0.13 & 1.55 & 0.21 & 3 & 9.59 & 3.19 & 4.58 & $5.02 \times 10^{-3}$ \\
\hline Residuals & 86 & 7.12 & 0.08 & & & 86 & 59.79 & 0.70 & & \\
\hline
\end{tabular}

lower for Incentive $(-11 \%)$ and Vision $(-14 \%)$. The degree of yield reduction was similar for Harper $(-15 \%)$, despite the observation that disease incidence and severity are significantly higher on this cultivar (Table 1). This indicates that stem striping is a poor indicator for predicting yield losses by V. longisporum.

Yield reductions for plots inoculated with the lower amount of inoculum were only significant for Quartz $(-17 \%)$ while the other cultivars did not suffer from measurable yield losses (Table 1). Across all cultivars, the median disease symptom severity negatively correlated to yield (Spearman's $\rho=-0.53, P=0.0001$ ). Similarly, the average disease incidence also correlated negatively with yield (Pearson's $r=-0.57, P=0$ ).

In contrast to the observations in 2016, the relationship between Verticillium stem striping symptoms and yield was different in the 2017 trial, since no significant differences between the different inoculum treatments were observed in yield for any of the cultivars assessed (Table 1). Verticillium stem striping symptoms, incidence (Pearson's $r=0.05, P=0.73$ ) and severity (Spearman's $\rho=0.09, P=0.52$ ) did not correlate with yield. Thus, although these data comprise only two cropping years, this indicates that the impact of $V$. longisporum is inconsistent despite the occurrence of abundant disease symptoms. This might be due to differences in host colonization patterns of $V$. longisporum between the two years (Siebold and von Tiedemann 2013). Different weather conditions between the two years may have an effect on yield reduction by $V$. longisporum. Elevated temperatures can enhance the $V$. longisporum colonization on oilseed rape, however, this effect is not consistently observed (Siebold and von Tiedemann 2013). Watering regimes were previously shown not to impact V. longisporum disease levels if grown under controlled greenhouse conditions (Lopisso et al. 2017).

The significant yield impact in harvest year 2016 showed that $V$. longisporum pathogen can be of economic significance in oilseed rape production. However, our inability to reproduce these yield reductions and the absence of significant oilseed rape yield reductions in a previous study indicate that significant yield reductions upon $V$. longisporum field inoculation only sporadically occur. As this study only comprises data from two consecutive cropping years, more field studies are needed to have a general assessment of the economic importance of Verticillium stem striping. Furthermore, to assess the impact of Verticillium stem striping under commercial conditions more accurately, propagule levels that are representative for $V$. longisporum infested agronomic soils should be used in future field trials, as we do not know if the inoculum amount applied on the soil in our study resembles the amount of propagules in commercially cultivated soils. To this end, species-specific primers can be used for $V$. longisporum DNA quantification in the soil (Debode et al. 2011; Tzelepis et al. 2017). In conclusion, the collection of yield data instead of striping symptoms in field trials would lead to a more genuine assessment of Verticillium stem striping resistance in oilseed rape cultivars, as we observed that striping symptoms can be untruthful predictors of final yielding outcomes.

Acknowledgements The authors would like to thank the Marie Curie Actions program of the European Commission that financially supports the research of J.R.L.D. Work in the laboratory of B.P.H.J.T. is supported by the Research Council Earth and Life Sciences (ALW) of the Netherlands Organization of Scientific Research (NWO). 
Compliance with ethical standards The authors declare that the research complies with ethical standards.

Conflict of interest The authors declare that they have no conflict of interest.

Open Access This article is distributed under the terms of the Creative Commons Attribution 4.0 International License (http:// creativecommons.org/licenses/by/4.0/), which permits unrestricted use, distribution, and reproduction in any medium, provided you give appropriate credit to the original author(s) and the source, provide a link to the Creative Commons license, and indicate if changes were made.

\section{References}

Debode, J., Van Poucke, K., França, S. C., Maes, M., Höfte, M., \& Heungens, K. (2011). Detection of multiple Verticillium species in soil using density flotation and real-time polymerase chain reaction. Plant Disease, 95, 1571-1580.

Depotter, J. R. L., Deketelaere, S., Inderbitzin, P., von Tiedemann, A., Höfte, M., Subbarao, K. V., et al. (2016). Verticillium longisporum, the invisible threat to oilseed rape and other brassicaceous plant hosts. Molecular Plant Pathology, 17, 1004-1016.

Depotter, J. R. L., Rodriguez-Moreno, L., Thomma, B. P. H. J., \& Wood, T. A. (2017a). The emerging British Verticillium longisporum population consists of aggressive Brassica pathogens. Phytopathology, 107, 1399-1405.

Depotter, J. R. L., Seidl, M. F., van den Berg, G. C. M., Thomma, B. P. H. J., \& Wood, T. A. (2017b). A distinct and genetically diverse lineage of the hybrid fungal pathogen Verticillium longisporum population causes stem striping in British oilseed rape. Environmental Microbiology, 19, 3997-4009.

Dunker, S., Keunecke, H., Steinbach, P., \& von Tiedemann, A. (2008). Impact of Verticillium longisporum on yield and morphology of winter oilseed rape (Brassica napus) in relation to systemic spread in the plant. Journal of Phytopathology, 156, 698-707.

Eynck, C., Koopmann, B., \& von Tiedemann, A. (2009). Identification of Brassica accessions with enhanced resistance to Verticillium longisporum under controlled and field conditions. Journal of Plant Diseases and Protection, 116, 63-72.

Gladders, P., Smith, J. A., Kirkpatrick, L., Clewes, E., Grant, C., Barbara, D., et al. (2011). First record of Verticillium wilt
(Verticillium longisporum) in winter oilseed rape in the UK. New Disease Reports, 23, 8.

Heale, J. B., \& Karapapa, V. K. (1999). The Verticillium threat to Canada's major oilseed crop: Canola. Canadian Journal of Plant Pathology, 21, 1-7.

Inderbitzin, P., \& Subbarao, K. V. (2014). Verticillium systematics and evolution: How confusion impedes Verticillium wilt management and how to resolve it. Phytopathology, 104, 564-574.

Inderbitzin, P., Bostock, R. M., Davis, R. M., Usami, T., Platt, H. W., \& Subbarao, K. V. (2011). Phylogenetics and taxonomy of the fungal vascular wilt pathogen Verticillium, with the descriptions of five new species. PLoS One, 6, e28341.

Koike, S. T., Subbarao, K. V., Davis, R. M., Gordon, T. R., \& Hubbard, J. C. (1994). Verticillium wilt of cauliflower in California. Plant Disease, 78, 1116-1121.

Lopisso, D. T., Knüfer, J., Koopmann, B., \& von Tiedemann, A. (2017). The vascular pathogen Verticillium longisporum does not affect water relations and plant responses to drought stress of its host, Brassica napus. Phytopathology, 107, 444- 454.

Novakazi, F., Inderbitzin, P., Sandoya, G., Hayes, R. J., von Tiedemann, A., Subbarao, K. V., et al. (2015). The three lineages of the diploid hybrid Verticillium longisporum differ in virulence and pathogenicity. Phytopathology, 105, 662-673.

Obermeier, C., Hossain, M. A., Snowdon, R., Knüfer, J., von Tiedemann, A., \& Friedt, W. (2013). Genetic analysis of phenylpropanoid metabolites associated with resistance against Verticillium longisporum in Brassica napus. Molecular Breeding, 31, 347-361.

Shaner, G., \& Finney, R. E. (1977). The effect of nitrogen fertilization on the expression of slow-mildewing resistance in Knox wheat. Phytopathology, 77, 1051-1056.

Siebold, M., \& von Tiedemann, A. (2013). Effects of experimental warming on fungal disease progress in oilseed rape. Global Change Biology, 19, 1736-1747.

Steventon, L. A., Fahleson, J., Hu, Q., \& Dixelius, C. (2002). Identification of the causal agent of Verticillium wilt of winter oilseed rape in Sweden, V. longisporum. Mycological Research, 106, 570-578.

Tzelepis, G., Bejai, S., Sattar, M. N., Schwelm, A., Ilbäck, J., Fogelqvist, J., et al. (2017). Detection of Verticillium species in Swedish soils using real-time PCR. Archives of Microbiology, 199, 1383-1389.

Zeise, K., \& von Tiedemann, A. (2002). Host specialization among vegetative compatibility groups of Verticillium dahliae in relation to Verticillium longisporum. Journal of Phytopathology, 150, 112-119. 